\title{
Histopathological Evaluation of Prevalent H. pylori Induced Gastro Intestinal Diseases According to Updated Sydney Classification
}

\author{
Samreen Soomro ${ }^{1}$, Tazeen Mustansir ${ }^{2}$, Talat Mirza ${ }^{3}$ \\ ${ }^{1}$ Faculty of Pharmacy, Northern Border University, NBU, Rafha, Saudi Arabia \\ ${ }^{2}$ Department of Pathology, Dow International Medical College, DIMC, Dow Diagnostic Reference and Research Lab, DDRRL, \\ Dow University of Health Sciences, DUHS, Karachi, Pakistan \\ ${ }^{3}$ Research and Postgraduates Studies, Ziauddin University, Karachi, Pakistan \\ Email: soomro.samreen@gmail.com
}

How to cite this paper: Soomro, S., Mustansir, T. and Mirza, T. (2019) Histopathological Evaluation of Prevalent H. pylori Induced Gastro Intestinal Diseases According to Updated Sydney Classification. Journal of Biosciences and Medicines, 7, 195-201.

https://doi.org/10.4236/jbm.2019.75021

Received: March 27, 2019

Accepted: May 24, 2019

Published: May 27, 2019

Copyright $\odot 2019$ by author(s) and Scientific Research Publishing Inc. This work is licensed under the Creative Commons Attribution International License (CC BY 4.0).

http://creativecommons.org/licenses/by/4.0/

\begin{abstract}
Objective: To determine the prevalence of $H$. pylori induced gastritis and its association with development of chronic inflammation and carcinoma with reference to updated Sydney classification, to ensure the proper diagnosis and timely treatment. Methods: This was a cross sectional study conducted at the Department of Pathology, Dow Diagnostic Reference and Research Laboratory for the year 2018. Analysis of about 726 gastric biopsy specimens was performed to identify the epidemiological status of the disease. On the other hand, 100 of the specimens were analysed according to Sydney classification to ensure the accurate diagnosis of the gastritis and related disorders. Out of 726 biopsy samples positive samples for carcinoma were investigated in detail histological features. Giemsa stain was used to visualize the h pylori bacteria and the sections of biopsies were subjected to haematoxylin and eosin staining for histological analysis. Results: Out of 727 cases, H. pylori was found in $492(67.8 \%)$ patients. About $1.79 \%$ (13) cases were identified as adenocarcinoma, $0.27 \%$ (2) squamous cell carcinoma and $0.13 \%$ (1) as B cell non Hodgkin lymphoma. Results of Sydney classification. The degree of chronic inflammatory activity was $57 \%$ mild; $38 \%$ moderate and $3 \%$ severe out of a total of $67 \%$ cases in an active phase of $\mathrm{Hp}$ gastritis. $\mathrm{Hp}$-associated gastritis was the most common form of chronic gastritis, without intestinal metaplasia and with mild activity. The association of $h$ pylori with mild inflammation and Neutrophilic infiltrates of the lamina propria, pits, or surface epithelium was statistically significant ( $\mathrm{p}$ value $<0.05$ ). Conclusion: The frequency of $H$. pylori infection is common in our population but the infection is mild to moderate and its Sydney classification reveals that early diagnosis may pre-
\end{abstract}


vent the later complication.

\section{Keywords}

Helicobacter pylori, Sydney Classification, Histology, Gastritis

\section{Introduction}

Helicobacter pylori (H. pylori) is the main etiological agent for antral gastritis, and plays a leading pathogenic role in duodenal ulcers and majority of non-NSAID gastric ulcers. There is high prevalence of $H$. pylori in the developing world where people have low socio-economic status and live in an unhygienic environment. Therefore, it is one of the most important causes or prerequisites of peptic and duodenal ulcer disease in Pakistani population [1] [2] [3]. A recent study revealed an early colonization/infection of infants with $H$. pylori and a prevalence of $67 \%$ at 9 months of age in a peri-urban community in Karachi, Pakistan [4]. In addition, some studies also revealed that gastritis among Pakistani population associated with pylori is estimated to be $51 \%-78 \%$ due to H. pylori infection [5]. Undiagnosed infection may lead to other associated diseases particularly there is a significant association between $H$. pylori infection and mucosa associated lymphoid tissue lymphomas (MALTomas) [6] and gastric cancer [5]. Interestingly, $H$. pylori could be present in a significant number of dyspeptic patients with endoscopically normal stomach [7] which may cause peptic ulcer disease. However, it is more common in duodenal ulcer than gastric ulcer and frequency of bacterial load increases with the increasing age [8]. Invasive tests have been considered as gold standard and timely diagnosis of $H$. pylo$r i$ infection is crucial for eradication of infection to prevent atrophic gastritis and GC which are consequences of peptic ulcer [9].

However current picture is quite different instead of being elimination of the disease the incidence of $\mathrm{H}$. pylori induced gastritis is getting higher, so there is a dire need to identify the current status of the prevalence of the disease in our country so that socioeconomic burden can be reduced. Therefore, objective of the current study is to audit the prevalence of $H$. pylori associated gastritis among Pakistani population with the severity of the disease and other associated diseases like carcinomas.

\section{Methods}

\subsection{Specimen Collection}

A total of 726 samples of Dyspeptic patients underwent upper gastrointestinal endoscopy in DUHS, Karachi, Pakistan and analysis of clinical features and epidemiological analysis was done. Out of 726 samples, 100 samples were selected for the details investigation according to Sydney grading. Therefor four biopsies were collected from each patient (two each from body and antrum of the stom- 
ach). The histopathological confirmation of $H$. pylori infection was accomplished by $\mathrm{H} \& \mathrm{E}$ and where required by Giemsa staining. The presence of $H$. pylori was graded as absent or present based on the Sydney system.

\subsection{Histology}

Giemsa stain was done to visualize $H$. pylori and biopsy specimens were fixed in $10 \%$ formalin embedded in paraffin wax before staining with Haematoxylin and eosin (H \& E) for histopathology [10]. The level of acute and chronic inflammation was measured according to an increase in lymphocytes and plasma cells in the lamina propria. H. pylori density on the basis of colonization was scored. Activity was scored by expansion of lamina propria pits and surface epithelium with neutrophilic polymorph infiltration. Atrophy was observed as the loss of inherent glandular tissue. Intestinal Metaplasia was classified according to their presence in individual biopsy specimens into grade 1 (focal) and grade 2 (occupying the entire width). All the pathologies were scored as none; mild; moderate and sever. For optimal histological evaluation, all gastric biopsy specimens included surface epithelium and muscularis mucosae.

\subsection{Statistical Analysis}

The statistical package for social science SPSS (Release 16, standard version, copyright ${ }^{\oplus}$ SPSS; 2007) was used for data analysis. The descriptive analysis was done for demographic and clinical features. Results were presented as mean for quantitative variables and number (percentage) for qualitative variables.

\section{Results}

\subsection{Clinical Features and Endoscopic Analysis}

The symptoms of the patients included abdominal pain $70 \%$, weight loss $17.5 \%$, vomiting $12.5 \%$, bloating $10 \%$, anaemia $17.5 \%$ and diarrhoea $15 \%$ epigastric pain $42.5 \%$, dyspepsia $27.5 \%$, and flatulence $10 \%$. The endoscopic findings of gastritis in patients with Non Ulcer dyspepsia (NUD) 50\%, gastric carcinoma in 3.5\%, gastric ulcer in $7.2 \%$ and duodenal ulcer in $10 \%$ was identified.

\subsection{Demographic and Epidemiological Analysis}

In current study about 726 samples were analysed and it was identifying on the basis of Giemsa stain that about $492(67.8 \%)$ having H. pylori associated gastritis whereas $234(32.2 \%)$ patients were having nonspecific gastritis. Out of 726 patients 58 (6\%) cases were of duodenal ulcer and only $16(2 \%)$ patients were diagnosed with carcinoma among which about $1.79 \%$ (13) cases were identified as adenocarcinoma, $0.27 \%$ (2) squamous cell carcinoma and $0.13 \%$ (1) as B cell non Hodgkin lymphoma.

The male to female was ratio was 1:1.02 for the prevalence of the disease. According to age group, the $H$. Pylori infection was more prevalent among middle age group $44.1 \%$ whereas it was least prevalent among elderly $21.1 \%$. 


\subsection{Morphological Variables According to Sydney Grading System of Chronic Gastritis}

The degree of chronic inflammatory activity was assessed and graded as follows: $57 \%$ mild; $38 \%$ moderate and $3 \%$ severe out of a total of $67 \%$ cases in an active phase of Hp gastritis (Table 1). Hp-associated gastritis was the most common form of chronic gastritis, while there was no association found between $H$. pylori infection and metaplasia. The gastric atrophy of lower antral mucosa was not found in $53.8 \%$ patients however the mild atrophy was noticed in $46.15 \%$ patients. The presence of $H$. pylori as significant pathological factor in mild cases was $84.6 \%$ however around 11.53 percent of cases have secondary reasons for gastritis other than $H$. pylori around $38 \%$ of population with gastritis showed no activity while $61.53 \%(50 \%+11.5 \%)$ showed mild to moderate Neutrophilic infiltrates of the lamina propria, pits, or surface epithelium (Table 1).

Table 1. Shows the Sydney grading classification on $H$. pylori associated gastritis.

\begin{tabular}{cccccc}
\hline \multirow{2}{*}{ S. No } & variables & \multicolumn{4}{c}{ \% of Sydney grading } \\
\cline { 3 - 6 } & & Nil & Mild & Moderate & Sever \\
\hline 1 & Chronic & 0 & 57.69 & 38.46 & 3.84 \\
2 & Inflammation & 38.46 & 50 & 11.53 & 0 \\
3 & Activity & 53.84 & 46.15 & 0 & 0 \\
4 & Atrophy & 100 & 0 & 0 & 0 \\
5 & $\begin{array}{c}\text { Intestinal } \\
\text { Metaplasia }\end{array}$ & & & & \\
\hline
\end{tabular}

Key of Variables' Grading that was used in this study listed below: 1. Chronic Inflammation $\rightarrow 5$ lymphocytes/hpf, >2 plasma cells/hpf, >5 lymphocytes/100 epithelial nuclei (The density of mononuclear cells in the lamina propria is graded in areas away from lymphoid follicles and their surrounding marginal zone of small lymphocytes). 2 . Activity $-<1 / 3=$ mild $2 / 3=$ moderate; $2 / 3=$ severe (expansion of lamina propria, pits, or surface epithelium with Neutrophilic infiltrates). 3. Atrophy-Mild, moderate, or severe (loss 3 to 4 glands). 4. Intestinal Metaplasia-Classified according to their presence in individual biopsy specimens into grade 1 (focal) and grade 2 (occupying the entire width). 5. H. pylori Density-Scattered orgs $<1 / 3$ of the surface $=$ mild; large clusters $/$ continuous layer $>2 / 3$ of surface $=$ severe intermediate numbers $=$ moderate colonization.

\section{Discussions}

The study of $H$. pylori virulence factor is important as it is associated with considerable morbidity and mortality. In Pakistan, infection with $H$. pylori is frequent among the local population and is acquired at an early age. prevalence of H. pylori infection varies strongly worldwide more than $80 \%-90 \%$ in developing countries [11]. The symptoms for $H$. pylori infection may vary from person to person and depending on chronicity as well as severity of infection. The most noticeable symptom in our population was found to be abdominal pain and epigastric pain followed by dyspepsia. Additionally, endoscopic findings of gastritis in patients revealed the highest percentage of non-ulcer dyspepsia. 
In the present study, we found that the total prevalence of $H$. pylori infection was $67.8 \%$ in this region, which was similar to the countrywide average reported previously in the year 2012 that is $74.4 \%$ [12]. Our study showed that there was no association between the $H$. pylori infection and sex. Previous studies showed that the prevalence of $H$. pylori infection increased with age, and the prevalence was lower in subjects younger than 20 years old [13], but in our study the prevalence in middle age is higher among other study groups.

$H$. pylori screening among the general population and immediate treatment has potentially reduced development of $H$. pylori associated complications and gastric cancer mortality [14]. Our data show a significant percentage of positive histopathological features such as neutrophil infiltration activity, density and chronic inflammation in cases of Hp gastritis. Since the of $H$. pylori increases the chances of developing adenocarcinoma and MALT lymphoma, early detection and eradication of $\mathrm{Hp}$ gastritis is of great importance for our patients for our patients in order to prevent the development of precancerous changes and GC. Although the number of patients with critical symptom of carcinoma was least prevalent in our population with total cases of $1.79 \%$ suffering with adenocarcinoma, squamous cell carcinoma and B cell non Hodgkin lymphoma.

The sites of the biopsy and Giemsa staining is crucial in diagnosis of $H$. pylori microbes and gastric atrophy. According to the Updated Sydney Classification System, biopsies should be taken from the mid antrum and mid body. Thus the Sydney scale was applied in our biological samples, since histological evaluation of Hp gastritis could be of great value in evaluating the treatment efficacy [15]. The degree of chronic inflammatory activity was assessed and graded as Sydney classification; the chronic inflammation was mild to moderate in our samples, along with mild activity of Neutrophilic infiltrates. Hp-associated gastritis was the most common form of chronic gastritis; while intestinal metaplasia was absent in our study cases. It was found to be a strong association of gastritis with the presence of $H$. pylori in biopsy specimens, contrast, reasonable number of patients may have secondary reasons for gastritis other than $H$. pylori as reported earlier [16].

Thus the Sydney system-based grading scale in comparison with routine assessment methods methods for population suffering from gastritis could be a tool to provide an objective histological evaluation. This might help in treatment efficacy of gastritis either related to H. Pylori or secondary reasons for gastritis [17] [18] [19].

\section{Conflicts of Interest}

The authors declare no conflicts of interest regarding the publication of this paper.

\section{References}

[1] Walsh, J.H. and Peterson, W.L. (1995) The Treatment of Helicobacter pylori for In- 
fection in the Management of Peptic Ulcer Disease. The New England Journal of Medicine, 333, 954-991. https://doi.org/10.1056/NEJM199510123331508

[2] Pounder, R.E. and Ng, D. (1995) The Prevalence of Helicobacter pylori Infection in Different Countries. Alimentary Pharmacology and Therapeutics, 9, 33-39.

[3] Hill, G., Majmudar, P., Shankaran, K., et al. (1994) Age Related Prevalence of Helicobacter pylori Antibodies in Indian Subjects. Indian Journal of Gastroenterology, 13, 92-94.

[4] Nizami, S.Q., Bhutta, Z.A., Weaver, L. and Preston, T. (2005) Helicobacter pylori Colonization in Infants in a Peri-Urban Community in Karachi, Pakistan. Journal of Pediatric Gastroenterology and Nutrition, 4, 191-194. https://doi.org/10.1097/01.mpg.0000172263.12920.6b

[5] Jafri, W., Yakoob, J., Abid, S., Siddiqui, S., Awan, S. and Nizami, S.Q. (2010) Helicobacter pylori Infection in Children: Population-Based Age Specific Prevalence and Risk Factors in a Developing Country. Acta Paediatrica, 99, 279-282. https://doi.org/10.1111/j.1651-2227.2009.01542.x

[6] Abbas, Z., Jafri, W., Khan, A.H. and Shah, M.A. (1998) Prevalence of Helicobacter pylori Antibodies in Endoscopy Personnel and Non-Medical Volunteers of Karachi. Journal-Pakistan Medical Association, 48, 201-202.

[7] Hassan, S.R. and Abbas, Z. (2007) Presence of Helicobacter pylori in Dyspeptic Patients with Endoscopically Normal Stomach. Pakistan Journal of Medical Sciences, 23, 335-339.

[8] Ghani, M.H., Ghouri, A.A., Hanif, R., Bux, H., Ahmed, S. and Humaira, M. (2007) Frequency of Helicobacter pylori in Patients with Peptic Ulcer Disease. Med Channel, 13, 60-65.

[9] Kokkola, A., Rautelin, H., Puolakkainen, P., Sipponen, P., Färkkilä, M., Haapiainen, R. and Kosunen, T.U. (2000) Diagnosis of Helicobacter pylori Infection in Patients with Atrophic Gastritis: Comparison of Histology, 13C-Urea Breath Test, and Serology. Scandinavian Journal of Gastroenterology, 35, 138-141. https://doi.org/10.1080/003655200750024290

[10] Mumtaz, K., Abid, S., Yakoob, J., Abbas, Z., Hamid, S., Islam, M., Shah, H.A. and Jafri, W. (2006) An Office-Based Serological Test for Detection of Current Helicobacter pylori Infection: Is It Useful? European Journal of Gastroenterology and Hepatology, 18, 85-88. https://doi.org/10.1097/00042737-200601000-00015

[11] Shi, R., Xu, S., Zhang, H., Ding, Y., Sun, G., Huang, X., Chen, X., Li, X., Yan, Z. and Zhang, G. (2008) Prevalence and Risk Factors for Helicobacter pylori Infection in Chinese Populations. Helicobacter, 13, 157-165. https://doi.org/10.1111/j.1523-5378.2008.00586.x

[12] Rasheed, F., Ahmad, T. and Bilal, R. (2012) Prevalence and Risk Factors of Helicobacter pylori Infection among Pakistani Population. Original Article, 28, 661-665.

[13] Malcolm, C.A., MacKay, W.G., Shepherd, A. and Weaver, L.T. (2004) Helicobacter pylori in Children Is Strongly Associated with Poverty. Scottish Medical Journal, 49, 136-138. https://doi.org/10.1177/003693300404900406

[14] Dixon, M.F., Genta, R.M., Yardley, J.H. and Correa, P. (1996) Classification and Grading of Gastritis: The Updated Sydney System. The American Journal of Surgical Pathology, 20, 1161-1181. https://doi.org/10.1097/00000478-199610000-00001

[15] Suzana, M.K., Skender, T., Emine, D.S., Halil, A., Vjollca, S.M., Agron, K., Sadushe, L., Labinot, S., Goneta, G. and Arijeta, P. (2009) Helicobacter pylori Gastritis Updated Sydney Classification Applied in Our Material. Journal of Natural Science, 
Biology and Medicine, 30, 45-60.

[16] El-Zimaity, H.M., Graham, D.Y., Al-Assi, M.T., Malaty, H., Karttunen, T.J., Graham, D.P., Huberman, R.M. and Genta, R.M. (1996) Interobserver Variation in the Histopathological Assessment of Helicobacter pylori Gastritis. Human Pathology, 27, 35-41. https://doi.org/10.1016/S0046-8177(96)90135-5

[17] Capelle, L.G., de Vries, A.C., Haringsma, J., Ter Borg, F., de Vries, R.A., Bruno, M.J., van Dekken, H., Meijer, J., van Grieken, N.C. and Kuipers, E.J. (2010) The Staging of Gastritis with the OLGA System by Using Intestinal Metaplasia as an Accurate Alternative for Atrophic Gastritis. Gastrointestinal Endoscopy, 71, 1150-1158. https://doi.org/10.1016/j.gie.2009.12.029

[18] Alhomsi, M.F. and Adeyemi, E.O. (1996) Grading Helicobacter pylori Gastritis in Dyspeptic Patients. Comparative Immunology, Microbiology and Infectious Diseases, 19, 147-154. https://doi.org/10.1016/0147-9571(95)00028-3

[19] Graham, D.Y., Nurgalieva, Z.Z., El-Zimaity, H.M., Opekun, A.R., Campos, A., Guerrero, L., Chavez, A. and Cardenas, V. (2006) Noninvasive versus Histologic Detection of Gastric Atrophy in a Hispanic Population in North America. Clinical Gastroenterology and Hepatology, 4, 306-314.

https://doi.org/10.1016/j.cgh.2005.11.003

\section{Abbriviations}

NUD = Non Ulcer Dyspepsia;

$\mathrm{GU}=$ Gastric Ulcer;

$\mathrm{DU}=$ Duodenal Ulcer;

$\mathrm{GC}=$ Gastric Carcinoma;

$\mathrm{Hp}=$ Helicobacter pylori. 